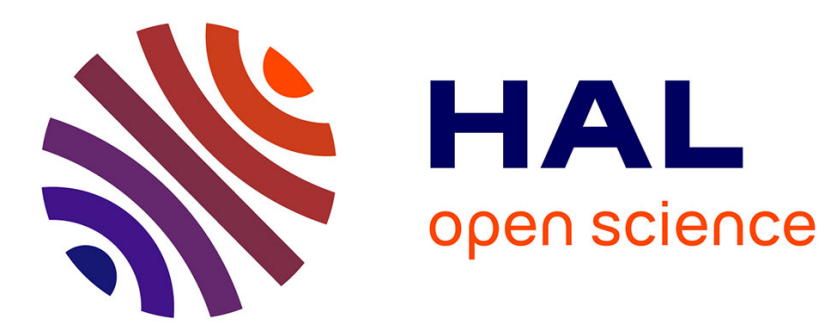

\title{
CPG-based Controllers can Generate Both Discrete and Rhythmic Movements
}

\author{
Melanie Jouaiti, Patrick Henaff
}

\section{To cite this version:}

Melanie Jouaiti, Patrick Henaff. CPG-based Controllers can Generate Both Discrete and Rhythmic Movements. IROS 2018 - IEEE/RSJ International Conference on Intelligent Robots and Systems, Oct 2018, Madrid, Spain. hal-01837189

\section{HAL Id: hal-01837189 \\ https://hal.science/hal-01837189}

Submitted on 12 Jul 2018

HAL is a multi-disciplinary open access archive for the deposit and dissemination of scientific research documents, whether they are published or not. The documents may come from teaching and research institutions in France or abroad, or from public or private research centers.
L'archive ouverte pluridisciplinaire HAL, est destinée au dépôt et à la diffusion de documents scientifiques de niveau recherche, publiés ou non, émanant des établissements d'enseignement et de recherche français ou étrangers, des laboratoires publics ou privés. 


\title{
CPG-based Controllers can Generate Both Discrete and Rhythmic Movements
}

\author{
Melanie Jouaiti ${ }^{1}$ and Patrick Henaff ${ }^{2}$
}

\begin{abstract}
Complex tasks require the combination of both discrete and rhythmic movements. Though scientists do not yet agree on the neural architecture involved in both types and in the transition from one to the other, the importance of having robot controllers able to behave rhythmically and discretely is universally recognized.

In this paper, a bio-inspired robot controller based on oscillating neurons is proposed to realize both discrete and rhythmic movements and easily transition from one to the other. It is shown that, under certain parameter conditions, the CPG controller behaves like a PID controller. In order to demonstrate the feasibility of controlling both discrete and rhythmic movements, the CPG is applied to the initiation of handshaking, namely, reach towards the human hand and start to shake it. Results show that this architecture is suitable for both discrete and rhythmic movements and can easily transition from one to the other.
\end{abstract}

\section{INTRODUCTION}

Discrete and rhythmic movements have been widely studied in motor neuroscience. Scientists have been wondering in which proportions movements are controlled by the central nervous system or attributed to intrinsic limb mechanics and how the brain can create complex movements with a combination of discrete and rhythmic movements and how those transitions are made.

Discrete movements are defined as singularly occurring events preceded and followed by a period without motion; rhythmic movements are continuous and recurring periodically. It was proposed that rhythmic movements might be a concatenation of discrete movements [1], [2] or on the contrary, discrete movements might be aborted rhythmic movements [3], [4]. In an attempt to identify the neural structures involved, a fMRI study by [5] revealed that rhythmic movements involve only a subset of the cortical activity observed in discrete movements.

Though those issues are not solved yet, it is obvious that complex tasks involve both discrete and rhythmic movements and should robots be able to reproduce those tasks, their controllers need to be able to realize both and easily transition from one mode to the other. Several such structures have already been proposed, they are mainly a combination of rhythmic and discrete pattern generators. [6] introduced a model associating the Hopf (rhythmic) and VITE (discrete) [7] models. In [8], the Matsuoka (rhythmic) [9] and VITE (discrete) models were brought together. [10], [11] proposed a model based solely on the Matsuoka oscillator but the

\footnotetext{
${ }^{1}$ Melanie Jouaiti is with Université de Lorraine, CNRS, Inria, LORIA, F-54000 Nancy, France melanie.jouaiti@loria.fr

${ }^{2}$ Patrick Hénaff is with Université de Lorraine, CNRS, Inria, LORIA, F-54000 Nancy, France patrick.henaffeloria.fr
}

discrete movement generated is merely the abortion of a rhythmic movement. As underlined by [12], the Matsuoka oscillator is intrinsically rhythmic and can't generate real discrete movements.

Few tasks are purely discrete or rhythmic in upper limbs movements. The discrete and rhythmic movements can be combined in two ways: the discrete phase aims at positioning the hand (reaching), followed by a rhythmic movement (basketball, cleaning, handshaking) or they can be both closely intertwined when the rhythmic movement undergoes a translation (sewing, piano playing). In this paper, we use handshaking as a benchmark. In gestural rhythmic communication, handshaking has an important and universally social function because it regulates and maintains human interactions [13], [14], [15], [16]. Different phases can be distinguished: preparation, contact, locking, synchronized rhythmic movement and withdrawal of the hands [17]. This work focuses mainly on the genesis of the handshake, i.e a robotic arm raises towards a hand and starts oscillating.

In this paper, a bio-inspired robot controller is proposed. It is based on oscillating neurons [18] able to realize both discrete and rhythmic movements and easily transition from one to the other. This controller is applied to the task of handshaking: the preparation, i.e. the hand raising towards the hand of the other partner, is a discrete movement, the whole contact phase is rhythmic until the withdrawal of the hand which is, again, discrete. It is shown that, under certain conditions, this controller behaves like a PID controller.

In section II, we introduce the robot controller, detail the CPG and show that it can behave as a PID controller under certain assumptions. Then section III presents the results of the CPG applied to the initiation of handshaking both in simulation and with a real robot. Section IV is dedicated to the conclusion and future works.

\section{Material AND Methods}

First, the method used to control the robot, then the CPG architecture used in this paper are introduced. Finally, it is demonstrated that, under certain conditions, this neuron behaves like a PID controller.

\section{A. The robot neuro-controller}

The distance between the gripper of the robotic arm and the human hand captured by a camera is the input of our CPG during the discrete phase. When the gripper grasps the human hand, it switches to rhythmic mode and starts oscillating. This paper is not interested in the human-robot interaction (this is part of an ongoing work), only in providing a 
framework for the transition discrete-rhythmic movement, so during the rhythmic phase, no input is provided to the CPG and the arm oscillates at its own intrinsic frequency. Three joints are controlled, two are responsible for movements in the sagittal plane, the third pans the frontal plane. The CPG output is the velocity command sent to the joint. Fig. 1 represents the sensori-motor control loop of the system.

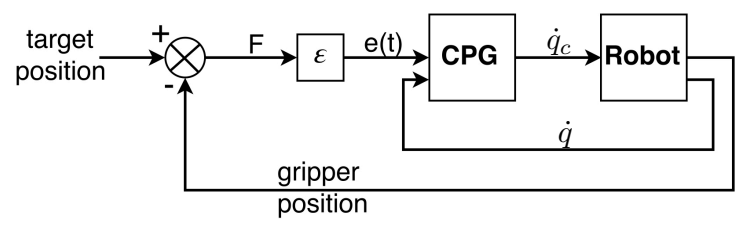

Fig. 1. CPG sensori-motor control loop. With $\dot{q}_{c}$ the command velocities sent to the joints, $\dot{q}$ the actual joint velocities.

\section{B. $C P G$ Architecture}

A CPG is a biological structure found in the spinal cord of vetebrates. Composed of a network of oscillatory neurons, it can generate a rhythmic signal without receiving any rhythmic input but which can be modulated by sensory feedbacks. The role of CPGs in walking has been proven and well studied and its implication in rhythmic upper limb movements is also strongly suspected [19], [20]. CPGs are based on a pair of two mutually inhibitory oscillating neurons, called half-center [21], controlling the extensor and flexor muscles. Non-linear CPGs, also called relaxation oscillators, can synchronize with an oscillatory input or with a coupled CPG, thus ensuring coordination.

An interesting bio-inspired model of half-center CPG for mammal locomotion was proposed by McCrea and Rybak [22]. The CPG is divided into two parts representing the extensor and flexor muscles and has four layers: Rhythm Generator, Pattern Formation, Sensory Neurons and Motoneurons. It also takes sensory feedback into account. While this model is widely used for locomotion [23], [24], [25], [26], very few works apply it to arm movements: to our knowledge, only [27] used it to study the reaching movement.

In this work, a model of CPG joint controller inspired by [22] is proposed (see Fig. 2, equations of neurons are given in the next section). The rhythmic layer is composed of Rowat-Selverston oscillating neurons (RG cells). The pattern formation layer is composed of interneurons (PF) and the motor layer is composed of motoneurons (MN). Feedbacks from the robot are applied to the CPG through sensory neurons ( $\mathrm{SN})$

\section{Mathematical Modeling}

For the rhythm generators, Rowat-Selverston cells are used. They are generalized versions of the Van der Pol oscillator [18] and can exhibit the four characteristic behaviors of a biological rhythmic neuron: endogenous bursting, plateau potential, post-inhibitory rebound and quiescence [28].

Let us recall the equations for the unforced form of the Rowat-Selverston model. For more details, refer to [18]:

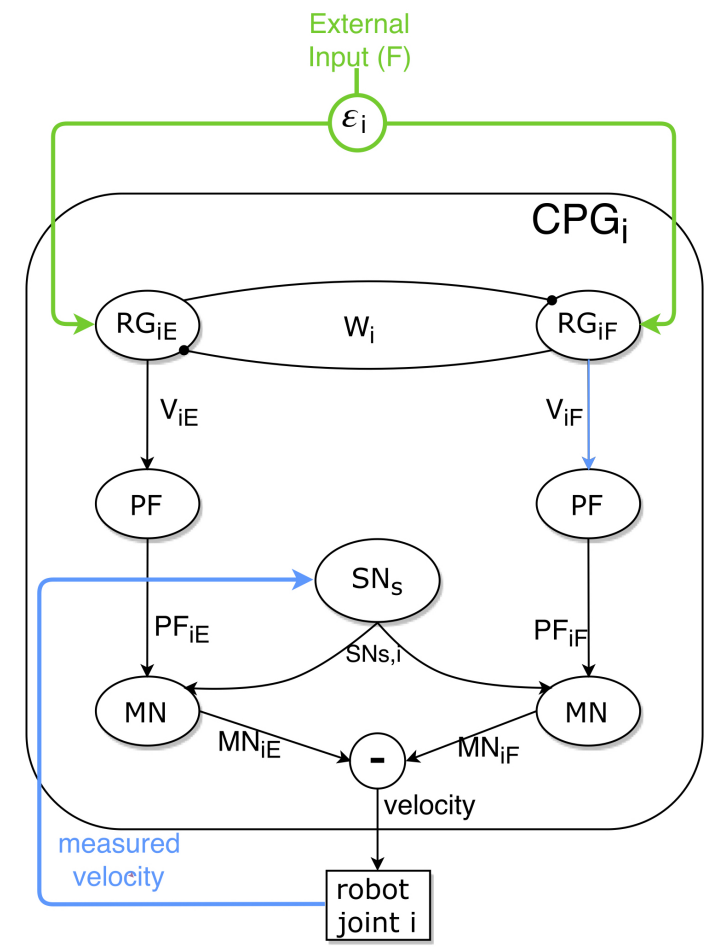

Fig. 2. Proposed CPG architecture inspired by [22]. Each CPG controls one joint. The CPGs are not coupled with each other.

$$
\begin{aligned}
& \tau_{m} \dot{V}+F(V)+q=0 \\
& F(V)=V-A_{f} \tanh \left(\frac{\sigma_{f}}{A_{f}}\right) \\
& \tau_{s} \dot{q}=-q+\sigma_{s} V
\end{aligned}
$$

with $\mathrm{V}$ the membrane potential and $\tau_{m}$ its time constant, $\mathrm{q}$ the slow current and $\tau_{s}$ its time constant. $F(V)$ is a Nshaped curve, whose degree and width are determined by $\sigma_{f}$ and $A_{f}$ respectively. For $\sigma_{f}=0, F(V)$ is linear, for $0<\sigma_{f}<1, F(V)$ has only point of inflexion and otherwise, it is $\mathrm{N}$-shaped. $\sigma_{s}$ is a gain. To be included in the proposed CPG architecture [22], equations of the rhythmic cells can be rewritten as (please refer to [29] for all the details):

$$
\begin{aligned}
\dot{V}_{i_{\{E, F\}}} & =y_{i_{\{E, F\}}}-W \frac{y_{i_{\{E, F\}}}}{1+e^{-4 y_{i_{\{F, E\}}}}}+\epsilon_{i_{\{E, F\}}} F_{i} \\
\dot{y}_{i_{\{E, F\}}} & = \\
& \left(\sigma_{f}-\frac{\tau_{m}}{\tau_{s}}-1-\sigma_{f} \tanh ^{2}\left(\frac{\sigma_{f}}{A_{f_{i}}} V_{i_{\{E, F\}}}\right)\right) \frac{y_{i_{\{E, F\}}}}{\tau_{m}} \\
& -\frac{1+\sigma_{s}}{\tau_{s} \tau_{m}} V_{i_{\{E, F\}}}+\frac{A_{f_{i_{\{E, F\}}}}}{\tau_{s} \tau_{m}} \tanh \left(\frac{\sigma_{f} V_{i_{\{E, F\}}}}{A_{f_{i_{\{E, F\}}}}}\right)
\end{aligned}
$$

With $i \in \mathbb{N}$, designating the joint id. $F$ is an external signal applied as the input of the CPG, $\epsilon$ a synaptic weight designed to scale the input and the term in $\mathrm{W}$ models the mutual inhibition between the rhythmic cells for the extensor and flexor.

Pattern Formation neuron PF, Sensory neuron SN and Motoneurons MN are defined as follows [29]: 


$$
\begin{aligned}
& P F\left(V_{i_{\{E, F\}}}\right)=P F_{i_{\{E, F\}}}=\frac{1}{1+e^{\frac{-V_{i_{\{E, F\}}}}{2}}} \\
& S N_{s}\left(v_{\text {mes }_{i}}\right)=S N_{i, s}=\frac{1}{1+e^{\alpha_{s} v_{i_{m e s}}}} \\
& M N\left(P F_{i_{\{E, F\}}}, S N_{i, s}\right)=M N_{i_{\{E, F\}}}= \\
& \frac{1}{1+e^{\alpha_{m}\left(P F_{i_{\{E, F\}}}-S N_{i, s}\right)}}
\end{aligned}
$$

with $\alpha_{s}=-0.061342, \alpha_{r}=3 . v_{\text {mes }_{i}}$ is the angular velocity measured for the given joint.

\section{The oscillating neuron can behave as a PID controller}

The great number of parameters available for the Rowat Selverston neuron allows us to tune its behavior and offers a wide range of possibilities. Their capacity to generate both rhythmic and discrete movements has already been exploited in [25]. In [18], it was determined that the neuron can't oscillate for $\sigma_{f}=0$, since $F(V)$ from equation (1) requires $\sigma_{f}>1+\frac{\tau_{m}}{\tau_{s}}$ to be sufficiently N-shaped. Let us apply this to equation (2) considering a single oscillating neuron and neglecting the inhibitory synapse $(W=0)$ :

$$
\begin{aligned}
& \dot{V}=y+\epsilon F \\
& \ddot{V}+\frac{\tau_{s}+\tau_{m}}{\tau_{s} \tau_{m}} \dot{V}+\frac{1+\sigma_{s}}{\tau_{s} \tau_{m}} V=0
\end{aligned}
$$

To control the CPG, the input will be the error in position of the gripper given a target position (Fig. 1). As such, the $\epsilon F$ term in equation (2) can be viewed as an error term $e(t)$, yielding:

$$
\begin{aligned}
& \dot{V}=y+e(t) \\
& \ddot{V}+\frac{\tau_{s}+\tau_{m}}{\tau_{s} \tau_{m}} \dot{V}+\frac{1+\sigma_{s}}{\tau_{s} \tau_{m}} V=0
\end{aligned}
$$

which is similar to the model of a second order system. Injecting the expression of $\dot{V}$ in equation (8):

$$
\dot{y}+\dot{e}(t)+\frac{\tau_{s}+\tau_{m}}{\tau_{s} \tau_{m}}(y+e(t))+\frac{1+\sigma_{s}}{\tau_{s} \tau_{m}} \int y+e(t) d t=0
$$

Separating the terms:

$$
\begin{aligned}
& \frac{\tau_{s}+\tau_{m}}{\tau_{s} \tau_{m}} y+\frac{1+\sigma_{s}}{\tau_{s} \tau_{m}} \int y d t+\dot{y}= \\
& \frac{\tau_{s}+\tau_{m}}{\tau_{s} \tau_{m}} e(t)+\frac{1+\sigma_{s}}{\tau_{s} \tau_{m}} \int e(t) d t+\dot{e}(t)
\end{aligned}
$$

Since $y=\dot{V}$ and assuming that the sigmoids act linearly when $\sigma_{f}=0, V \approx \alpha \dot{q}$, equation (10) can be rewritten as :

$$
\alpha(a \ddot{q}+b \dot{q}+\dddot{q})=a e(t)+b \int e(t) d t+\dot{e}(t)
$$

With $a=\frac{\tau_{s}+\tau_{m}}{\tau_{s} \tau_{m}}, b=\frac{1+\sigma_{s}}{\tau_{s} \tau_{m}}, \dot{q}$ the joint velocities, $\ddot{q}$ the joint accelerations and $q$ could be identified as the jerk. Equation (11) can be rewritten as follows in which $\dot{q}$ is generated by the CPG controller and is thus the joint command velocity:

$$
\dot{q}=\frac{1}{\alpha b}(\underbrace{a e(t)+b \int e(t) d t+\dot{e}(t)}_{P} \underbrace{-a \ddot{q}}_{D}-\dddot{q})
$$

In term D of equation (12), $\ddot{q}$ represents the dynamical behavior of the robot which is often represented by the following dynamical equation:

$$
A(q) \ddot{q}+C(q, \dot{q}) \dot{q}+G(q)=\Gamma
$$

which yields:

$$
\ddot{q}=A(q)^{-1}(\Gamma-C(q, \dot{q}) \dot{q}-G(q))
$$

Part $\mathrm{P}$ of equation (12) is very similar to the PID equation:

$$
u(t)=K_{p} e(t)+K_{i} \int e(t) d t+K_{d} \dot{e}(t)
$$

This demonstrates that the command computed by the CPG takes into account both the robot dynamics and a classical control rule, such as the PID. This shows that, for some parameter values, the neuron behaves like a PID controller, which can be observed in simulation (see Fig. 3). The greater $\epsilon$, the more the system oscillates. So, $\epsilon$ acts as a gain for the error $e(t)$ in the sensori-motor control loop.
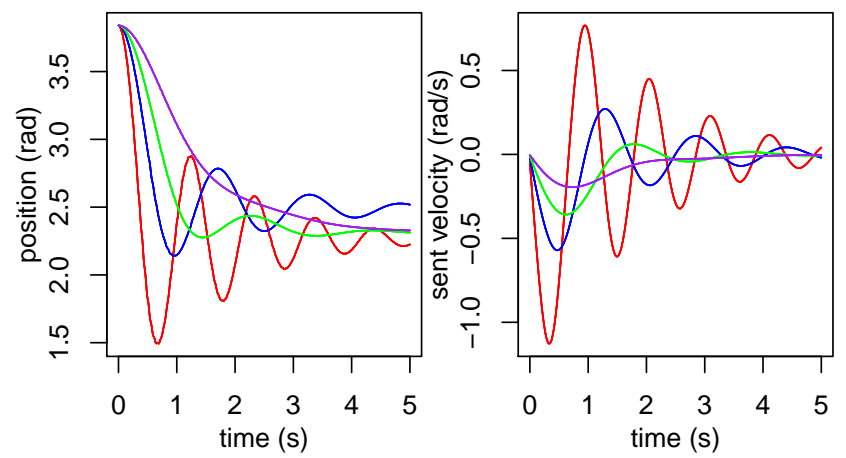

Fig. 3. Evolution of the position (left) of a joint controlled by our CPG in discrete mode and the output of the CPG (right) for various values of $\epsilon$. In red, $\epsilon=5$; in blue, $\epsilon=1.9$; in green, $\epsilon=1$; in purple, $\epsilon=0.5$.

\section{RESULTS}

Simulations were run with a Kinova robotic arm. First the simulated results and then the results with the actual robot are presented. For all experiments, the following CPG time constants are chosen such that the CPG intrinsic frequency can be set around $2 \mathrm{~Hz}$ (usual handshaking frequency according to [30]): $\tau_{m}=0.35, \tau_{s}=3.5$. 

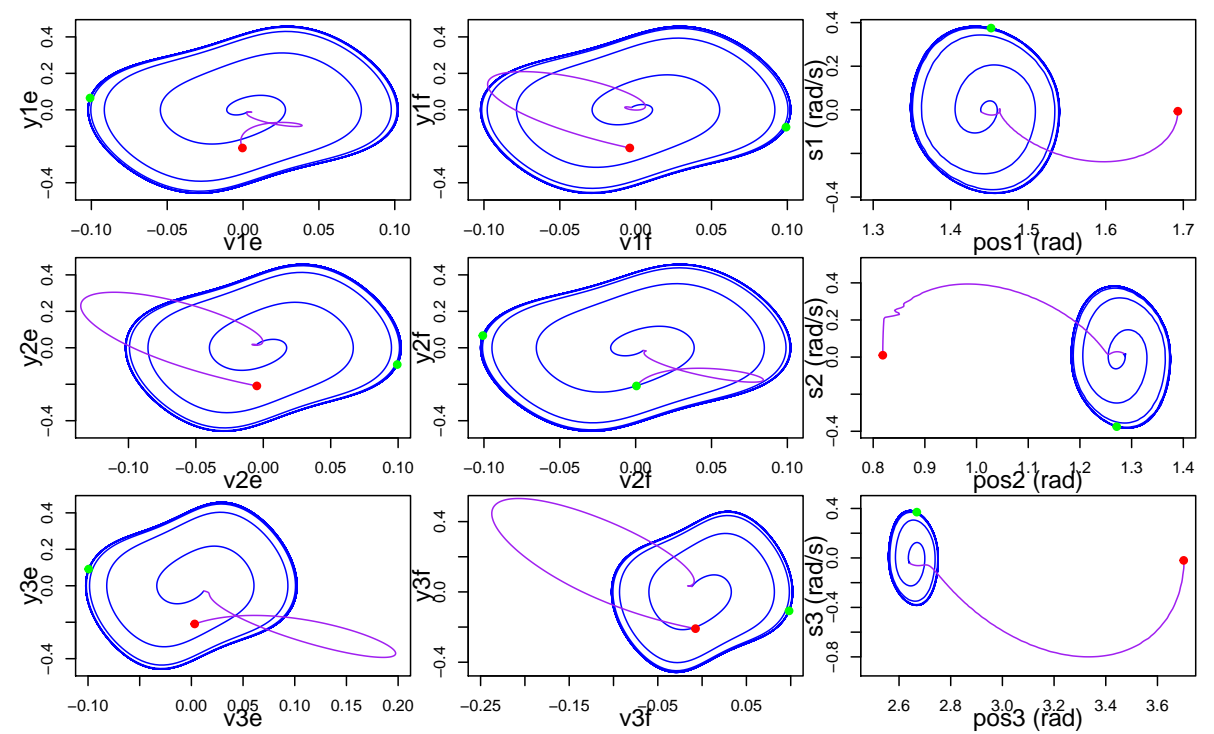

Fig. 4. Phase portrait of the output of the CPG for the extensor and flexor (left and middle) and of the joint position (right) for each joint (top to bottom, joint 1 to 3 respectively) in simulation. The red dot indicates the start and the green one, the end of the experiment. In purple, the discrete phase; in blue, the rhythmic phase.

\section{A. Simulated Results}

Experiments were conducted with a Kinova robotic arm in the simulator V-REP. For convenience purposes, the robotic arm aims to grab a ball instead of a hand (due to the difficulties of simulating a human hand with V-Rep). The simulation provides us with the $3 \mathrm{D}$ position of the target ball and the gripper (see Fig. 5). From there, computing the distance between both is trivial. Three joints are controlled: joint 1 aims to reduce the error distance $d y$ in the $y$ axis, joint 2 and 3, the error $d z$ in the $z$ axis.

Fig. 6 represents the CPG input, output and angular position for each joint during the simulation. Two phases can be distinguished.

Discrete phase (from $\mathbf{t}=\mathbf{0} \mathbf{s}$ to $\mathbf{t}=\mathbf{3 . 5 8} \mathrm{s}$ ): At the beginning of the experiment, the robotic arm is at rest and in discrete mode $\left(\sigma_{f}=0, \sigma_{s}=10, \epsilon_{1}=0.7, \epsilon_{2}=0.4\right.$, $\left.\epsilon_{3}=0.8, A_{f}=0.05\right)$. Each iteration, the distance error between the gripper and the ball is computed and fed to the CPGs which control the three joints. The discrete phase lasts until the gripper grasps the ball. By then, every CPG input is zero, meaning that each joint has reached its target.

Rhythmic phase (from $\mathbf{t}=3.58 \mathbf{s}$ to $\mathbf{t}=\mathbf{2 0}$ s): If $d z$ and $d y$ are under a given threshold, the ball moves in the $\mathrm{x}$ direction towards the gripper. When the distance in the $\mathrm{x}$ axis is sufficiently small, the gripper closes the robot switches to
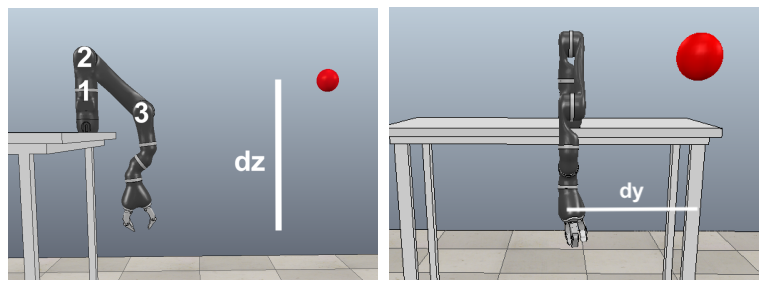

Fig. 5. Simulation setup at the beginning. The arm is at rest and the ball to grab in front of it.

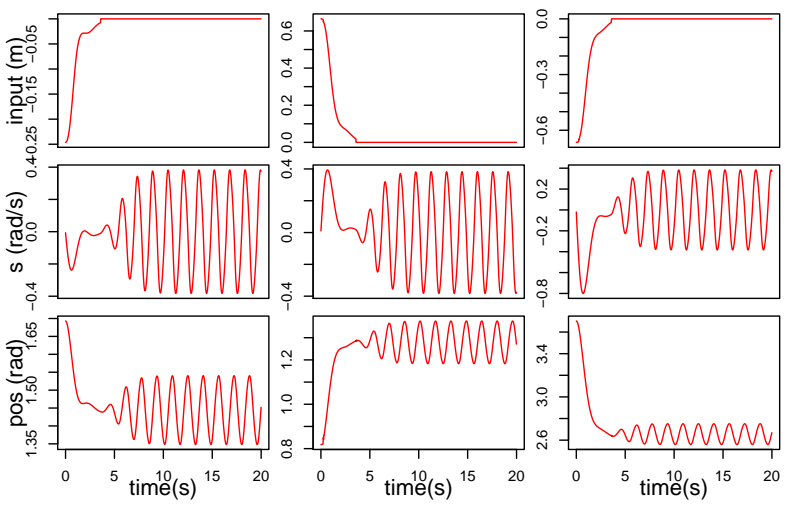

Fig. 6. Evolution of the input, output of the CPG (joint velocity sent) and the position of the joint controlled for each joint with the simulated robot. On the left, joint 1 ; in the middle, joint 2 ; on the right, joint 3 .

the rhythmic mode by setting $\sigma_{f}=2$ and $\sigma_{s}=20$ ( $A_{f}=$ $0.1)$. Note that while $\sigma_{s}$ has to be below a given value for the CPG to generate discrete movements, it could stay at 10 in the rhythmic mode, it is merely changed to obtain faster oscillations. Similarly, $A_{f}$ is modified for display purposes. In the rhythmic phase, the CPG receives no input and simply oscillates at its own intrinsic frequency $(0.6 \mathrm{~Hz})$ until the end of the interaction.

Both phases can also be clearly identified in the phase portraits (see Fig. 4). It should be noted that the discrete and rhythmic phases, as well as the CPG transition from PID controller to non-linear oscillator are stable. Once the arm enters the rhythmic mode, limit cycles appear.

\section{B. Experimental Results}

Experiments were conducted with the Kinova Mico robot (see Fig. 7). The robot gripper was replaced with a static 3D printed hand. The position of the robot hand is determined with the forward kinematic model of the robot according 


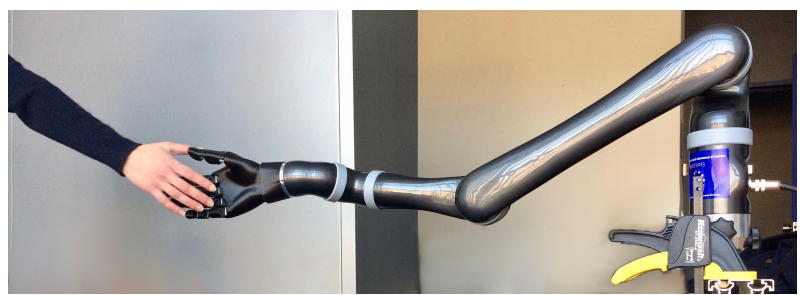

Fig. 7. Experimental setup. Mico robot with its hand reaching towards the human hand.

to a chosen referential. The robot is coupled to a webcam and the hand to grasp is detected thanks to a simple color segmentation algorithm implemented in OpenCV 3.4. In order to make the detection easier, the human partner wears a glove of distinctive color. The position of the human hand is estimated in the $z$ and $y$ plane only, given the detection results. From there, the experimental setup is the same as before: the arm raises towards the hand, reducing the error $d y$ and $d z$, the human grasps the robot hand and the latter starts oscillating.

Fig. 8 represents the CPG input, output and angular position for each joint during the experimentation. It can be divided into two phases.

Discrete phase (from $\mathbf{t}=\mathbf{0} \mathrm{s}$ to $\mathbf{t}=\mathbf{1 . 3} \mathrm{s}$ ): At the beginning of the experiment, the robotic arm is at rest and in discrete mode $\left(\sigma_{f}=0, \sigma_{s}=10, \epsilon_{1}=0.5, \epsilon_{2}=0.5, \epsilon_{3}=0.3\right.$, $\left.A_{f}=0.05\right)$. It raises towards the ball until $d y$ and $d z$ reach a given threshold.
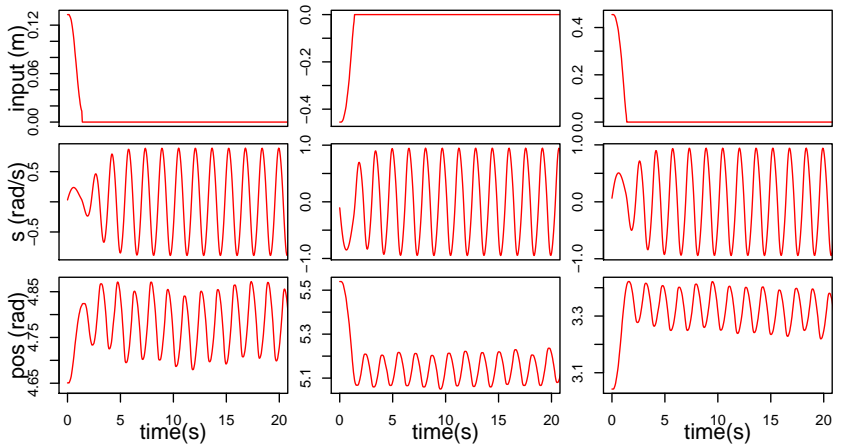

Fig. 8. Evolution of the input, output of the CPG (joint velocity sent) and the position of the joint controlled for each joint with the real robot. On the left, joint 1 ; in the middle joint 2 ; on the right, joint 3.

Rhythmic phase (from $\mathbf{t}=1.3 \mathrm{~s}$ to $\mathbf{t}=\mathbf{2 0} \mathrm{s}$ ): $d y$ and $d z$ being under the given threshold triggers the switch to discrete movement by setting $\sigma_{f}=2$ and $\sigma_{s}=20\left(A_{f}=0.1\right)$. Each joint oscillates at its own intrinsic frequency $(0.6 \mathrm{~Hz})$ since it receives no input. The oscillations are maintained until the end of the interaction.

Both phases can be once again clearly identified in the phase portraits (see Fig. 9). The discrete and rhythmic phases, as well as the CPG transition from PID controller to non-linear oscillator are stable. Once the arm enters the rhythmic mode, limit cycles appear. The discrete phase appears quite different from the simulation. This may come from the robot PID controller. Indeed, the real robot has a build-in PID controller but the V-Rep robot doesn't when controlled in velocity mode, so an additional PID controller had to be implemented. Differences in results may come from implementation variations.

\section{CONCLUSION}

This paper proposed a bio-inspired robot controller based on the Rowat-Selverston neuron able to realize both discrete and rhythmic movements and easily transition from one to the other by simply changing one parameter. It was shown that, under certain parameter conditions, this neuron can behave like a PID controller. Genesis of handshaking (reach towards a human hand and then shake it) allowed us to illustrate the capacities of the controller. It was demonstrated, both in simulation and with a real Kinova robot, that our architecture is suitable for both discrete and rhythmic movements and can smoothly transition from one to the other. While some currently available controllers can already achieve this, they require combining rhythmic and discrete pattern generators. However, in our controller, the pattern generator can switch easily from one mode to the other, simply by changing a single parameter.

From a neuroscience point of view, $\sigma_{f}$ could be modulated by descending signals from higher centers or neuromodulators determining when and how the CPG switches between discrete and rhythmic movements. For instance, for the handshaking gesture in robotics, the change in $\sigma_{f}$ might be triggered by a sensory neuron if the robot is equipped with force or contact sensors, that is $\sigma_{f}=1$ if contact is occurring, $\sigma_{f}=0$, otherwise. CPGs able to generate discrete movements, as well as rhythmic movements, present a particular interest in humanoid robotics, notably for discrete movements such as reaching, complex movements which are both discrete and rhythmic (handshaking) and reflexive responses to stimuli (perturbation while walking). Robust CPGs with such capacities would be invaluable in humanrobot interactions, enabling complex and natural interactions.

Besides, the presence of acceleration and jerk in the PID equation seems to indicate that the neuron behaves as a PID controller, but not only. It could be more than a simple PID controller, taking the robot dynamics into account as well. We will investigate the role of these extra terms.

Next steps in this work involve studying the robustness to perturbations of the controller and comparing its performance with other hybrid methods. We will also experiment handshaking as a whole with a robot equipped with an articulated artificial hand able to grasp, and study the synchronization with the human partner and the withdrawal gesture.

\section{ACKNOWLEDGMENT}

We thank Adrien Guénard for his technical help with the robotic arm and with the fabrication of the artificial hand.

\section{REFERENCES}

[1] L. Baratto, P. Morasso, and R. Zaccaria, "Complex motor patterns: Walking," in Advances in Psychology, vol. 33, pp. 60-81, Elsevier, 1986.

[2] A. Feldman, "Superposition of motor programs-i. rhythmic forearm movements in man," Neuroscience, vol. 5, no. 1, pp. 81-90, 1980. 

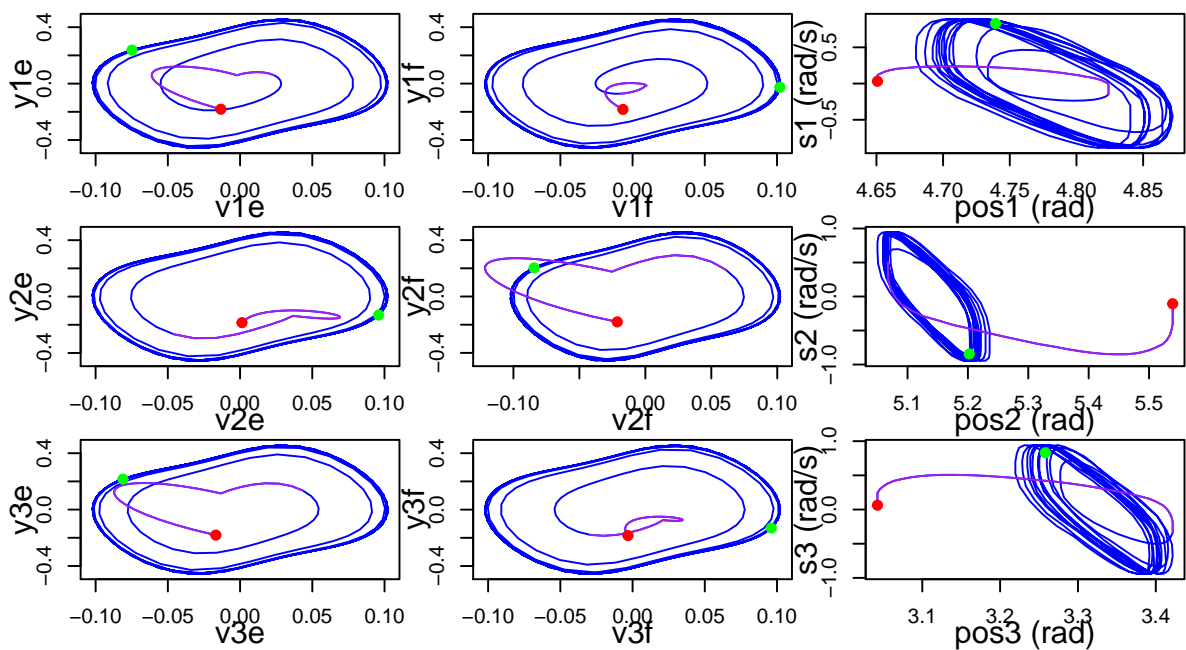

Fig. 9. Phase portrait of the output of the CPG for the extensor and flexor (left and middle) and of the joint position (right) for each joint (top to bottom, joint 1 to 3 respectively) with the real robot. The red dot indicates the start and the green one, the end of the experiment. In purple, the discrete phase; in blue, the rhythmic phase.

[3] D. Mottet and R. J. Bootsma, "The dynamics of goal-directed rhythmical aiming," Biological cybernetics, vol. 80, no. 4, pp. 235-245, 1999.

[4] Y. Guiard, "On fitts's and hooke's laws: Simple harmonic movement in upper-limb cyclical aiming," Acta psychologica, vol. 82, no. 1-3, pp. 139-159, 1993.

[5] S. Schaal, D. Sternad, R. Osu, and M. Kawato, "Rhythmic arm movement is not discrete," Nature neuroscience, vol. 7, no. 10, p. 1136, 2004.

[6] S. Degallier, L. Righetti, S. Gay, and A. Ijspeert, "Toward simple control for complex, autonomous robotic applications: combining discrete and rhythmic motor primitives," Autonomous Robots, vol. 31, no. 2-3, pp. 155-181, 2011.

[7] D. Bullock and S. Grossberg, "The vite model: a neural command circuit for generating arm and articulator trajectories," Dynamic patterns in complex systems, pp. 305-326, 1988.

[8] D. Sternad, W. J. Dean, and S. Schaal, "Interaction of rhythmic and discrete pattern generators in single-joint movements," Human Movement Science, vol. 19, no. 4, pp. 627-664, 2000.

[9] K. Matsuoka, "Sustained oscillations generated by mutually inhibiting neurons with adaptation," Biological cybernetics, vol. 52, no. 6, pp. 367-376, 1985.

[10] A. de Rugy and D. Sternad, "Interaction between discrete and rhythmic movements: reaction time and phase of discrete movement initiation during oscillatory movements," Brain Research, vol. 994, no. 2, pp. 160-174, 2003.

[11] D. Sternad, "Towards a unified theory of rhythmic and discrete movements-behavioral, modeling and imaging results," in Coordination: Neural, behavioral and social dynamics, pp. 105-133, Springer, 2008.

[12] S. Degallier and A. Ijspeert, "Modeling discrete and rhythmic movements through motor primitives: a review," Biological cybernetics, vol. 103, no. 4, pp. 319-338, 2010.

[13] D. Schiffrin, "Handwork as ceremony: the case of the handshake," Semiotica, vol. 12, no. 3, pp. 189-202, 1974.

[14] P. M. Hall and D. A. Spencer Hall, "The handshake as interaction," Semiotica, vol. 45, no. 3-4, pp. 249-264, 1983.

[15] F. J. Bernieri and K. N. Petty, "The influence of handshakes on first impression accuracy," Social Influence, vol. 6, no. 2, pp. 78-87, 2011.

[16] E. Giannopoulos, Z. Wang, A. Peer, M. Buss, and M. Slater, "Comparison of people's responses to real and virtual handshakes within a virtual environment," Brain research bulletin, vol. 85, no. 5, pp. 276282, 2011

[17] E. J. Walker, W. F. Bischof, and A. Kingstone, "Take my hand: The temporal and spatial coordination of handshaking," in Joint Action Meeting of the Cognitive Science Society, 2013.
[18] P. F. Rowat and A. I. Selverston, "Modeling the gastric mill central pattern generator of the lobster with a relaxation-oscillator network," Journal of neurophysiology, vol. 70, no. 3, pp. 1030-1053, 1993.

[19] S. Schaal, "Dynamic movement primitives-a framework for motor control in humans and humanoid robotics," in Adaptive motion of animals and machines, pp. 261-280, Springer, 2006.

[20] E. P. Zehr, T. J. Carroll, R. Chua, D. F. Collins, A. Frigon, C. Haridas, S. R. Hundza, and A. K. Thompson, "Possible contributions of cpg activity to the control of rhythmic human arm movement," Canadian journal of physiology and pharmacology, vol. 82, no. 8-9, pp. 556$568,2004$.

[21] S. Grillner and P. Wallen, "Central pattern generators for locomotion, with special reference to vertebrates," Annual review of neuroscience, vol. 8, no. 1, pp. 233-261, 1985.

[22] I. A. Rybak, N. A. Shevtsova, M. Lafreniere-Roula, and D. A. McCrea, "Modelling spinal circuitry involved in locomotor pattern generation: insights from deletions during fictive locomotion," The Journal of physiology, vol. 577, no. 2, pp. 617-639, 2006.

[23] J. Nassour, P. Hénaff, F. Benouezdou, and G. Cheng, "Multi-layered multi-pattern cpg for adaptive locomotion of humanoid robots, biological cybernetics," Biological cybernetics, vol. 108, no. 3, pp. 291-303, 2014.

[24] E. Amrollah and P. Henaff, "On the role of sensory feedbacks in rowat-selverston cpg to improve robot legged locomotion," Frontiers in neurorobotics, vol. 4, 2010.

[25] S. Debnath, J. Nassour, and G. Cheng, "Learning diverse motor patterns with a single multi-layered multi-pattern cpg for a humanoid robot," in Humanoid Robots (Humanoids), 2014 14th IEEE-RAS International Conference on, pp. 1016-1021, IEEE, 2014.

[26] S. M. Danner, S. D. Wilshin, N. A. Shevtsova, and I. A. Rybak, "Central control of interlimb coordination and speed-dependent gait expression in quadrupeds," The Journal of physiology, vol. 594, no. 23, pp. 6947-6967, 2016.

[27] W. W. Teka, K. C. Hamade, W. H. Barnett, T. Kim, S. N. Markin, I. A. Rybak, and Y. I. Molkov, "From the motor cortex to the movement and back again," PloS one, vol. 12, no. 6, p. e0179288, 2017.

[28] E. Marder and D. Bucher, "Central pattern generators and the control of rhythmic movements," Current biology, vol. 11, no. 23, pp. R986R996, 2001.

[29] M. Jouaiti, L. Caron, and P. Henaff, "Hebbian plasticity in cpg controllers facilitates self-synchronization for human-robot handshaking," Frontiers in Neurorobotics, 2018.

[30] G. Tagne, P. Hénaff, and N. Gregori, "Measurement and analysis of physical parameters of the handshake between two persons according to simple social contexts," in Intelligent Robots and Systems (IROS), 2016 IEEE/RSJ International Conference on, pp. 674-679, 2016. 